José Gomes Bezerra Filho'

Lígia Regina Franco Sansigolo Kerr Pontes'

Daniel de Lima Miná'

Maurício Lima Barreto"

\section{Mortalidade infantil e condições sociodemográficas no Ceará, em 1991 e 2000}

Departamento de Saúde Comunitária. Universidade Federal do Ceará. Fortaleza CE, Brasil

" Instituto de Saúde Coletiva. Universidade Federal da Bahia. Salvador, BA, Brasil

Correspondência | Correspondence: José Gomes Bezerra Filho Faculdade de Medicina da UFC

Departamento de Saúde Comunitária R. Prof. Costa Mendes $16085^{\circ}$ Andar Bairro Rodolfo Teófilo

60416-200 Fortaleza, CE, Brasil

E-mail: gomes@ufc.br

\section{Infant mortality and sociodemographic conditions in Ceará, Brazil, 1991 and 2000}

\section{RESUMO}

OBJETIVO: Analisar os modelos explicativos ecológicos para a taxa de mortalidade infantil no Ceará, em dois períodos distintos.

MÉTODOS: Estudo ecológico transversal de dois anos censitários, 1991 e 2000, a partir de informações desagregadas por municípios do Ceará. Foram utilizadas as estimativas da taxa de mortalidade infantil do Instituto de Pesquisas Econômicas Aplicadas. Para os demais indicadores foram utilizadas diferentes fontes do Sistema de Informação de Saúde. Os principais fatores de risco foram encontrados empregando-se regressão linear múltipla.

RESULTADOS: Para 1991, as variáveis preditoras da mortalidade infantil $(\mathrm{R} 2=0,3575)$ nos municípios foram: proporção de residências pequenas $(\beta=0,0043 ; \rho=0,010)$, de pessoas que vivem em domicílios com água encanada $(\beta=-0,0029 ; \rho=0,024)$, de crianças de dez a 14 anos que trabalham $(\beta=0,0049$; $\rho=0,017)$, de alfabetizados $(\beta=-0,0062 ; \rho=0,031)$, taxa de urbanização $(\beta=0,0032 ; \rho=0,004)$, taxa de fecundidade total $(\beta=0,0351 ; \rho=0,024)$, chefes de família com renda mensal menor que meio salário mínimo $(\beta=0,0056 ; \rho=0,000)$. Em 2000, os possíveis determinantes $(\mathrm{R} 2=0,3236)$ foram: proporção de crianças menores de dois anos desnutridas $(\beta=0,0064 ; \rho=0,024)$, de imóveis com esgotamento sanitário adequado $(\beta=-0,0024 ; \rho=0,010)$, de despesa com recursos humanos da saúde em relação à despesa total em saúde $(\beta=-0,0024 ; \rho=0,027)$, de valor da produção vegetal em relação ao total do estado $(\beta=-0,1090 ; \rho=0,001)$, de mulheres alfabetizadas $(\beta=-0,0068 ; \rho=0,044)$, intensidade da pobreza $(\beta=0,0065 ; \rho=0,002)$ e índice de envelhecimento $(\beta=-0,0100 ; \rho=0,006)$.

CONCLUSÕES: Embora as variáveis não tenham sido exatamente as mesmas para os anos, percebeu-se tendência de mudança dos determinantes da mortalidade infantil, excetuando-se os indicadores de educação, renda e saneamento. A queda generalizada da fecundidade resultou na perda de seu poder descriminante, sendo substituída pelo índice de envelhecimento. Outra tendência observada foi a substituição de variáveis demográficas por indicadores de assistência à saúde.

DESCRITORES: Mortalidade infantil. Fatores socioeconômicos. Fatores de risco. Modelos lineares. 


\section{ABSTRACT}

OBJECTIVE: To assess ecological models to describe infant mortality rate in Ceara (Northeastern Brazil) in two different periods of time.

METHODS: This was a cross-sectional ecologic study of two years, 1991 and 2000, using non-matching information per municipalities. Estimates on the infant mortality rate of the Instituto de Pesquisas Econômicas Aplicadas (Institute of Applied Economic Research) have been used. For the remaining indicators different sources of the System of Health Information were used. The main risk factors were assessed using multiple linear regression.

RESULTS: In 1991, the variables that predicted infant mortality rate $(\mathrm{R} 2=0.3575)$ were: small houses $(\beta=0.0043 ; \rho=0.010)$, proportion of inhabitants with tap water in the household $(\beta=-0.0029 ; \rho=0.024)$, urbanization rate $(\beta=0.0032 ; \rho=0.004)$, fecundity rate $(\beta=0.0351 ; \rho=0.024)$, the proportion of children working at $10-14$ years $(\beta=0.0049 ; \rho=0.017)$, proportion of families with income $<1 / 2$ minimum wage $(\beta=0.0056 ; \rho=0.000)$, that can read and write ( $\beta=-0.0062 ; \rho=0.031)$. In the year 2000 , the following possible determinants were identified $(\mathrm{R} 2=0.3236)$ : the proportion of children $<2$ years of age with malnutrition $(\beta=0.0064 ; \rho=0.024)$, proportion of households with adequate sanitation $(\beta=-0.0024 ; \rho=0.010)$, proportion of women who could read and write $(\beta=-0.0068 ; \rho=0.044)$, expenses on health human resources regarding total health expenses $(\beta=-0.0024 ; \rho=0.027)$, proportion of the value of the vegetal production in relation to the total of the state $(\beta=-0.1090 ; \rho=0.001)$, intensity of poverty $(\beta=0.0065 ; \rho=0.002)$, and ageing index $(\beta=-0.0100 ; \rho=0.006)$.

CONCLUSIONS: Although the variables have not been exactly the same for the evaluated period, determiners of infant mortality have been changing, except for indicators of education, income and sanitation. The overall decrease in fecundity led to a reduction in its discriminating power, and it was replaced by the ageing index. Another tendency observed was the replace of several demographic variables by health care indicators.

\section{KEY WORDS: Infant mortality. Socioeconomic factors. Risk factors.} Linear models.

\section{INTRODUÇÃO}

A taxa de mortalidade infantil (TMI) reflete, de maneira geral, os níveis de saúde e de desenvolvimento socioeconômico de uma determinada área, sendo considerada um dos mais importantes indicadores epidemiológicos utilizados internacionalmente. ${ }^{10}$

Em países em desenvolvimento, como os da África Central, a TMI atinge média de 113 óbitos de crianças menores de um ano por mil nascidos vivos (nv), seguidos dos países asiáticos, cuja taxa global é de 55 óbitos por mil nv. A TMI da Europa é de nove óbitos por mil nv e na América do Norte, alcança o patamar de sete óbitos por mil nv. AAmérica do Sul é detentora do valor médio de 31 óbitos por mil nv. ${ }^{17}$
No ano de 2000, a TMI para o Brasil foi estimada 28,3 por mil nv, ${ }^{*}$ mas observa-se uma expressiva desigualdade entre os seus estados. Esse fato tem provocado o interesse de diversos pesquisadores e instituições de saúde na busca dos determinantes dessas disparidades. ${ }^{6}$ Embora nenhum estado brasileiro tenha apresentado aumento da mortalidade infantil nos últimos dez anos, 11 encontram-se acima da média nacional, dentre os quais nove pertencem à região Nordeste e dois, à região Norte. ${ }^{19}$

No Ceará, foi observada tendência de declínio da TMI no período de 1991 a 2000, quando variou de 66,8 para 39,8 por mil nv, representando uma queda de $40,4 \%$ **

* Ministério da Saúde. Departamento de Informática do Sistema Único de Saúde. Disponível em www.datasus.gov.br. Brasília; 2003.

** Ministério da Saúde. Informações de Saúde. Coeficiente de mortalidade infantil por ano segundo regiões e unidades da Federação. Brasil, 1989 a 1998. 2000. [acesso em 17/9/2007]. Disponível em http://www.tabnet.datasus.gov.br/cgi/mortinf/mibr.htm 
Todavia, em 2000, essa taxa foi superior à do Brasil (28,3 por mil nv) e à de todos os estados das demais regiões.* Apesar de não se dispor de informações sobre nv do início da década de 90 , a mortalidade infantil representava $30,4 \%$ e $69,6 \%$ do total de óbitos de menores de um ano, respectivamente, segundo componentes neonatal (menores de 28 dias) e pós-neonatal (28 dias a 11 meses) para o Ceará em 1991. Em 2000, essas mesmas cifras perfaziam $41 \%$ e $59 \%$ da mortalidade infantil. As taxas elevadas nesses segmentos traduzem, no primeiro, a inadequada assistência ao pré-natal, ao parto e ao recém-nascido, e no segundo, a precariedade das condições socioeconômicas e de saúde da mãe.*

A redução da mortalidade infantil é ainda um grande desafio no País. Apesar da importante queda registrada na última década, decorrente principalmente da redução da mortalidade pós-neonatal, as cifras ainda são elevadas. Há uma estagnação da mortalidade neonatal nas regiões mais desenvolvidas e um aumento relativo nas regiões e populações mais vulneráveis, conseqüência da redução da mortalidade infantil pós-neonatal. Essa situação é agravada quando se reconhece que em mais de $90 \%$ dessas mortes de menores de um ano poderiam ser evitadas por ações de prevenção, diagnóstico e tratamento precoces ou por meio de parcerias com outros setores.

É sabido que inúmeros nascimentos ocorrem sem o devido registro, principalmente nos municípios do interior do estado. Esses nascimentos são normalmente resultantes de partos domiciliares, portanto, não incluídos nos registros do Sistema de Informações sobre Nascidos Vivos (Sinasc) ou nas estatísticas do Registro Civil. Espera-se, que com os altos índices de cobertura do Programa de Saúde da Família (PSF) no Ceará, haja um descenso na subnotificação dos nascimentos. ${ }^{19}$

Levando-se em consideração as mudanças políticas, econômicas e assistenciais do final da década de 80 e durante a década de 90 , é plausível supor que tenha ocorrido alteração nos fatores associados à TMI no Ceará, fruto das mudanças sucedidas nessas décadas. O objetivo do presente trabalho foi discutir os modelos explicativos ecológicos para a TMI no Ceará em dois períodos distintos: 1991 e 2000.

\section{MÉTODOS}

Trata-se de um estudo ecológico transversal, de dois anos censitários, a partir de informações desagregadas por municípios do Ceará. O ano 1991 abrangeu todos os 178 municípios do Ceará, e em 2000, todos os 184 municípios.
As TMI de 1991 e 2000 utilizadas foram estimadas pelo Instituto de Pesquisas Econômicas Aplicadas (IPEA), em virtude da baixa cobertura do Sistema de Informações sobre Mortalidade no Ceará no início da década de 90. Para obtenção dos demais indicadores foram utilizadas diferentes fontes, constituintes do Sistema de Informação de Saúde do Ministério da Saúde, por meio do Departamento de Informática (Datasus). Outras fontes pesquisadas foram o Instituto Brasileiro de Geografia e Estatística (IBGE), Instituto de Pesquisa e Estratégia Econômica do Ceará (IPECE), Secretaria Estadual de Saúde do Ceará (SESA-CE) e Programa de Desenvolvimento das Nações Unidas (UNDP).

Foram utilizadas as seguintes variáveis:

- Indicador epidemiológico: taxa de mortalidade infantil (IPEA).

- Indicadores demográficos: taxa de urbanização (IPECE), densidade demográfica (IBGE), fecundidade total (IPEA), índice de envelhecimento e razão de dependência (IBGE).

- Indicadores de atenção à saúde, infra-estrutura e recursos humanos nos serviços de saúde: cobertura vacinal (Datasus), indicadores de amamentação e desnutrição infantil (Datasus e SESA-CE), leitos hospitalares (Datasus), médicos por habitantes (IPECE e Datasus), equipamentos e consultas de pré-natal por habitantes (Datasus).

- Indicadores socioeconômicos:

- Educação: proporção de crianças de sete a 14 anos de idade analfabetas proporção de crianças de sete a 14 anos de idade fora da escola, taxa de alfabetização de adultos, número médio de anos de estudo das pessoas de 25 anos de idade ou mais (IPEA); proporção de mulheres alfabetizadas e proporção de mulheres com menos de oito anos de estudo (IPECE); e componente para educação do Índice de Desenvolvimento Humano (IPEA e UNDP).

- Indicadores de habitação e saneamento: proporção de residências pequenas ( 2 ou menos cômodos), percentual da população com acesso a serviços de abastecimento público de água, esgoto, coleta de lixo (IPEA e IPECE); acesso à rede de energia e proporção de pessoas que vivem em domicílios com telefone (IPECE).

- Renda e produção: proporção de recursos financeiros dirigidos à saúde do município (Datasus); proporção de chefes de domicílios por rendimento em salários mínimos (IPECE e

* Ministério da Saúde. Departamento de Informática do Sistema Único de Saúde. Disponível em www.datasus.gov.br. Brasília; 2003. 
IBGE); proporção de crianças de dez a 14 anos de idade que trabalham (IPEA); proporção do valor da produção vegetal em relação ao total do estado, proporção do consumo de energia rural, industrial e comercial sobre consumo total do município, produto interno bruto per capita (IPECE); intensidade da pobreza (linha de $\mathrm{R} \$ 37,75)$ e Índice de Desenvolvimento Humano-M Renda (metodologia 2000) (IPEA e UNDP).

Foram empregadas as análises de regressão linear simples e múltipla para obter os coeficientes de regressão que relacionam cada variável no modelo com a TMI. Segundo Szwarcwald et al (2002), ${ }^{21}$ o coeficiente angular da regressão é considerado o melhor indicador para medir desigualdade em saúde quando as variáveis de nível de saúde e de nível socioeconômico podem ser expressas quantitativamente.

A construção dos modelos foi iniciada pela análise univariada, observando-se os pressupostos da regressão, particularmente, no que diz respeito à relação de linearidade entre o desfecho e as variáveis independentes. A partir dessa análise, aqueles indicadores cuja regressão sobre a TMI apresentava significância menor que 0,25 foram introduzidos no modelo de regressão linear múltipla. ${ }^{9}$ Para a regressão linear múltipla, foi aplicada a transformação log-neperiana na variável dependente como forma de normalizar a distribuição dos resíduos.

A estimativa dos parâmetros foi produzida de acordo com o método dos mínimos quadrados, seleção backward. Os melhores preditores foram escolhidos avaliando-se suas significâncias no modelo pelo teste de Wald, sendo de 0,05 o nível para preservação no modelo definitivo. ${ }^{9} \mathrm{O}$ modelo final foi selecionado pelo coeficiente de determinação $\left(\mathrm{R}^{2}\right)$ e pela normalidade dos seus resíduos. A metodologia descrita foi implementada utilizando os programas estatísticos Stata 7.0 e SPSS 10.

Além dos modelos para discussão de seus possíveis determinantes, foi realizado o diagnóstico de colinearidade por meio dos fatores de inflação da variação (VIF) para variáveis individuais de acordo com o critério de Hamilton (2003). ${ }^{8}$

De forma geral os modelos propostos são derivados das proposituras de Mosley ${ }^{14}$ (1988) e Arroyo et $\mathrm{al}^{2}$ (1988) que incorporavam variáveis sociais e biológicas ou mais recentes, determinantes distais e proximais para explicar o comportamento da mortalidade infantil.

\section{RESULTADOS}

A Tabela 1 apresenta o valor mínimo e máximo, a média e o desvio-padrão de todas as variáveis estudadas. A TMI obtida pela média dos municípios declinou de
70,69 para 47,27 óbitos por mil nv. Ainda assim, todos os municípios apresentaram taxas superiores a 25 por mil nv ao final do período, dos quais $35 \%$ apresentaram valores superiores a 50 por mil nv.

Dentre as variáveis de atenção à saúde, a cobertura vacinal no ano de 1991 tinha seu valor mínimo de $21,0 \%$ e média de $72,8 \%$, aumentando no ano de 2000 para $62,7 \%$ e $102,3 \%$, respectivamente, o que revela melhoria no período. A cobertura máxima apresentada para o ano de 2000 ultrapassou 100\% em diversos municípios. A explicação encontrada para o fato é a de que alguns municípios, por atenderem em regime de livre demanda, cobrem a população de municípios vizinhos e/ou a população em idade vacinal ter sido subestimada pelo IBGE.

Com relação às variáveis de saneamento, a proporção de domicílios urbanos e rurais com água canalizada aumentou, obtendo média de 21,5\% em 1991 e 40,7\% em 2000. A coleta de lixo em domicílios urbanos teve aumento na média da cobertura de 38,2\% em 1991 para $66,5 \%$ em 2000. O esgotamento sanitário em domicílios urbanos, apesar de melhorar sua abrangência (de 3,0\% em 1991 para $14,8 \%$ em 2000), ainda apresenta valor muito abaixo do necessário para caracterizar um bom nível sanitário.

Dentre os indicadores demográficos, destacou-se a taxa de fecundidade total, representando o número médio de filhos tidos pelas mulheres em seu período fértil; a média entre os municípios declinou de 4,59 para 3,37 no período, acompanhando a tendência nacional.

Dentre os indicadores econômicos, observou-se melhoria na proporção de chefes de família com renda mensal menor que meio salário mínimo, declinando a média de 33,2\% em 1991 para 16,5\% em 2000. No entanto, o indicador intensidade da pobreza (linha $R \$ 37,75=1 / 4$ salário mínimo) mostrou aumento de 45,2\% em 1991 para 55,1\% em 2000 (salário mínimo em 2000, de R\$ 151,00, equivalente a aproximadamente US\$ 77.00).

Frente aos indicadores educacionais, observou-se uma melhoria da proporção de mulheres alfabetizadas (média em 1991 de 60,0\% e em 2000 de 73,5\%), embora o Ceará apresente uma das maiores taxas de analfabetismo em nível nacional $(24,8 \%)$.

O logaritmo neperiano da TMI de 1991 foi mais bem explicado $\left(\mathrm{R}^{2}=0,3575\right)$ pelas seguintes co-variáveis: proporção de residências pequenas $(\beta=0,0043$; $\rho=0,010)$, de pessoas que vivem em domicílios com água encanada $(\beta=-0,0029 ; \rho=0,024)$, de crianças de 10 a 14 anos que trabalham $(\beta=0,0049 ; \rho=0,017)$, de chefes de família com renda mensal menor que $1 / 2$ salário míni$\operatorname{mo}(\beta=0,0056 ; \rho=0,000)$ e de alfabetizados $(\beta=-0,0062$; $\rho=0,031)$, taxa de urbanização $(\beta=0,0032 ; \rho=0,004)$, taxa de fecundidade total $(\beta=0,0351 ; \rho=0,024)$. 
Tabela 1. Análise descritiva da taxa de mortalidade infantil e indicadores socioeconômicos, demográficos e de atenção à saúde. Ceará, 1991 e 2000.

\begin{tabular}{|c|c|c|c|c|c|}
\hline \multirow{2}{*}{ Variável } & \multirow{2}{*}{ Unidade } & \multicolumn{2}{|c|}{1991} & \multicolumn{2}{|c|}{2000} \\
\hline & & Mínimo & Máximo & Mínimo & Máximo \\
\hline \multicolumn{6}{|l|}{ Epidemiológica } \\
\hline Taxa de mortalidade infantil* & Por $1000 \mathrm{NV}$ & 47,1 & 108,9 & 26,9 & 81,6 \\
\hline \multicolumn{6}{|l|}{ Atenção à saúde, recursos humanos e infraestrutura } \\
\hline Cobertura vacinal em $<1$ ano & Por 100 & 21,0 & 97,0 & 64,2 & 126,1 \\
\hline$\%$ de gestantes com 4 ou mais consultas pré-natal & Por 100 & 3,0 & 93,0 & 44,9 & 96,5 \\
\hline Número de médicos por mil hab. & Por 1000 hab & 0,0 & 3,1 & 0,1 & 4,3 \\
\hline Médicos do PSF por jornada** & Unidade & & & 0,0 & 30,0 \\
\hline Pediatra por jornada por mil nascidos vivos** & Unidade & & & 0,00 & 31,4 \\
\hline$\%$ de crianças $<4$ meses acompanhadas PACS/PSF só mamando & Por 100 & 0,0 & 62,0 & 26,8 & 84,8 \\
\hline$\%$ de menores de 1 ano desnutridos** & Por 100 & & & 3,3 & 33,2 \\
\hline$\%$ de menores de 2 anos desnutridas** & Por 100 & & & 0,0 & 25,9 \\
\hline Leitos hospitalares por mil hab.** & Por 1000 hab & & & 0,0 & 10,6 \\
\hline Presença ou ausência de salas de parto** & Sim/não & & & 0,0 & 1,0 \\
\hline Presença ou ausência de incubadora** & Sim/não & & & 0,0 & 1,0 \\
\hline \multicolumn{6}{|l|}{ Condições de habitação e saneamento } \\
\hline$\%$ de residências pequenas** & Por 100 & 8,7 & 60,2 & & \\
\hline \% de pessoas que vivem em domicílios com água canalizada & Por 100 & 0,3 & 74,0 & 2,2 & 88,5 \\
\hline$\%$ de domicílios urbanos com coleta de lixo & Por 100 & 0,0 & 88,5 & 13,5 & 95,1 \\
\hline$\%$ de pessoas que vivem em domicílios urbanos com inst. esgoto adequada & Por 100 & 0,0 & 49,9 & 0,1 & 81,1 \\
\hline$\%$ de pessoas que vivem em domicílios com energia elétrica e geladeira & Por 100 & 2,9 & 71,2 & 11,8 & 86,8 \\
\hline$\%$ de pessoas que vivem em domicílios com telefone & Por 100 & 0,2 & 24,8 & 1,8 & 55,2 \\
\hline \multicolumn{6}{|l|}{ Indicadores demográficos } \\
\hline Taxa de urbanização & Por 100 & 11,4 & 100,0 & 17,4 & 100,0 \\
\hline Razão de dependência & Por 100 & 61,9 & 108,2 & 52,6 & 92,9 \\
\hline Taxa de fecundidade total & Por 1 mulher & 2,5 & 7,8 & 2,2 & 4,9 \\
\hline Índice de envelhecimento & Por 100 & 6,3 & 29,4 & 10,3 & 37,5 \\
\hline \multicolumn{6}{|l|}{ Indicadores de renda e produção } \\
\hline Intensidade da pobreza: linha de $\mathrm{R} \$ 37,75$ & $\mathrm{R} \$$ & 32,7 & 61,5 & 38,3 & 73,6 \\
\hline Renda per capita & $\mathrm{R} \$$ & 27,3 & 232,4 & 46,4 & 306,7 \\
\hline Índice de Desenvolvimento Humano-M Renda & & 0,3 & 0,7 & 0,4 & 0,7 \\
\hline \% de chefes de domicílio com rendimento > 1 salário mínimo** & Por 100 & & & 13,8 & 67,2 \\
\hline$\%$ de chefes de família com renda mensal menor de $1 / 2 \mathrm{sm}$ (urbano) & Por 100 & 12,8 & 70,4 & 1,5 & 36,6 \\
\hline$\%$ consumo de energia indust. e comer. sobre consumo total do município** & Por 100 & & & 1,9 & 80,8 \\
\hline$\%$ do consumo de energia rural sobre o total do município** & Por 100 & & & 0,2 & 59,3 \\
\hline \% do valor da produção vegetal em relação ao total do estado** & Por 100 & & & 0,0 & 2,4 \\
\hline \% desp. com pessoal em relação a desp. total em saúde do município** & Por 100 & & & 4,1 & 78,5 \\
\hline \% das transf. do SUS em relação a despesa total em saúde do município** & Por 100 & & & 21,8 & 94,3 \\
\hline$\%$ de crianças 10 a 14 anos que trabalham & Por 100 & 2,8 & 41,2 & 2,7 & 32,1 \\
\hline \multicolumn{6}{|l|}{ Indicadores educacionais } \\
\hline$\%$ de crianças de 7 a 14 anos de idade analfabetas & Por 100 & 21,9 & 79,5 & 11,9 & 49,9 \\
\hline$\%$ de mulheres com 10 anos ou mais de idade alfabetizadas & Por 100 & 38,3 & 85,2 & 57,2 & 90,7 \\
\hline$\%$ mulheres com 10 anos ou mais de idade e menos de 8 anos de estudo & Por 100 & 65,3 & 99,0 & 54,8 & 89,2 \\
\hline$\%$ de alfabetizados*** & Por 100 & 21,4 & 69,3 & 39,0 & 77,3 \\
\hline Índice de Desenvolvimento Humano-M Educação & & 0,3 & 0,8 & 0,6 & 0,9 \\
\hline
\end{tabular}

* Fonte: Instituto de Pesquisa Econômica Aplicada - IPEA

** Variáveis disponíveis apenas para o ano de 2000

*** Variáveis disponíveis apenas para o ano de 1991 
Tabela 2. Descrição do modelo de regressão linear múltipla com base nos possíveis determinantes socioeconômicos e demográficos da taxa mortalidade infantil. Ceará, 1991.

\begin{tabular}{|c|c|c|c|c|}
\hline $\begin{array}{l}\text { Logaritmo neperiano da taxa de mortalidade } \\
\text { infantil* }\end{array}$ & $\begin{array}{l}\text { Coeficiente de } \\
\text { regressão } \beta\end{array}$ & $\begin{array}{l}\text { Coeficiente de } \\
\text { regressão } \\
\text { padronizado } \beta^{\prime}\end{array}$ & $\begin{array}{l}\text { Significância } \\
\text { de } \beta\end{array}$ & $\begin{array}{c}\text { Intervalo de } \\
\text { confiança de } \beta(95 \%)\end{array}$ \\
\hline Constante & 3,8960 & & 0,000 & 3,$523 ; 4,269$ \\
\hline Proporção de residências pequenas & 0,0043 & 0,183 & 0,010 & 0,$001 ; 0,008$ \\
\hline $\begin{array}{l}\text { Proporção de pessoas que vivem em domicílios } \\
\text { com água encanada }\end{array}$ & $-0,0029$ & $-0,222$ & 0,024 & $-0,005 ; 0,000$ \\
\hline Taxa de urbanização & 0,0032 & 0,311 & 0,004 & 0,$001 ; 0,005$ \\
\hline Taxa de fecundidade total & 0,0351 & 0,172 & 0,024 & 0,$005 ; 0,065$ \\
\hline $\begin{array}{l}\text { Proporção de crianças de } 10 \text { a } 14 \text { anos que } \\
\text { trabalham }\end{array}$ & 0,0049 & 0,169 & 0,017 & 0,$001 ; 0,009$ \\
\hline $\begin{array}{l}\text { Proporção de chefes de família com renda } \\
\text { mensal menor de } 1 / 2 \text { salário mínimo }\end{array}$ & 0,0056 & 0,321 & 0,000 & 0,$003 ; 0,008$ \\
\hline Proporção de alfabetizados & $-0,0062$ & $-0,228$ & 0,031 & $-0,012 ;-0,001$ \\
\hline
\end{tabular}

* Fonte: IPEA

$\mathrm{N}=178 \quad \mathrm{~F}=13,51 \quad \mathrm{R}^{2}=0,3575$

Tabela 3. Descrição do modelo de regressão linear múltipla com base nos possíveis determinantes socioeconômicos e demográficos da taxa mortalidade infantil. Ceará, 2000.

\begin{tabular}{|c|c|c|c|c|}
\hline $\begin{array}{l}\text { Logaritmo neperiano da taxa de mortalidade } \\
\text { infantil* }\end{array}$ & $\begin{array}{l}\text { Coeficiente de } \\
\text { regressão } \beta\end{array}$ & $\begin{array}{l}\text { Coeficiente de } \\
\text { regressão } \\
\text { padronizado } \beta^{\prime}\end{array}$ & $\begin{array}{l}\text { Significância } \\
\text { de } \beta\end{array}$ & $\begin{array}{c}\text { Intervalo de } \\
\text { confiança de } \beta(95 \%)\end{array}$ \\
\hline Constante & 4,2520 & & 0,000 & 3,$658 ; 4,846$ \\
\hline $\begin{array}{l}\text { Proporção de crianças menores de } 2 \text { anos } \\
\text { desnutridas }\end{array}$ & 0,0064 & 0,146 & 0,024 & 0,$001 ; 0,012$ \\
\hline $\begin{array}{l}\text { Proporção de imóveis com esgotamento } \\
\text { sanitário adequado }\end{array}$ & $-0,0024$ & $-0,182$ & 0,010 & $-0,004 ;-0,001$ \\
\hline Índice de envelhecimento & $-0,0100$ & $-0,180$ & 0,006 & $-0,017 ;-0,003$ \\
\hline $\begin{array}{l}\text { Proporção de despesa com pessoal em relação } \\
\text { à despesa total em saúde }\end{array}$ & $-0,0024$ & $-0,148$ & 0,027 & $-0,005 ; 0,000$ \\
\hline $\begin{array}{l}\text { Proporção do valor da produção vegetal em } \\
\text { relação ao total do estado }\end{array}$ & $-0,1090$ & $-0,221$ & 0,001 & $-0,172 ;-0,047$ \\
\hline Intensidade da pobreza & 0,0065 & 0,210 & 0,002 & 0,$002 ; 0,011$ \\
\hline Proporção de mulheres alfabetizadas & $-0,0068$ & $-0,154$ & 0,044 & $-0,013 ; 0,000$ \\
\hline
\end{tabular}

* Fonte: IPEA

$N=182 \quad F=11,89 \quad R^{2}=0,3236$

O logaritmo neperiano da TMI de 2000 foi mais bem modelado $\left(\mathrm{R}^{2}=0,3236\right)$ pelos seguintes fatores: proporção de crianças menores de dois anos desnutridas $(\beta=0,0064 ; \rho=0,024)$, de imóveis com esgotamento sanitário adequado $(\beta=-0,0024 ; \rho=0,010)$, da despesa com pessoal em relação à despesa total em saúde ( $\beta=-0,0024 ; \rho=0,027)$, do valor da produção vegetal em relação ao total do estado $(\beta=-0,1090 ; \rho=0,001)$ e de mulheres alfabetizadas $(\beta=-0,0068 ; \rho=0,044)$; intensidade da pobreza $(\beta=0,0065 ; \rho=0,002)$, índice de envelhecimento $(\beta=-0,0100 ; \rho=0,006)$.

\section{DISCUSSÃO}

A principal limitação da análise ecológica é esperar que inferências causais do efeito ecológico reflitam o efeito biológico do nível individual. ${ }^{12}$ Outro problema refere-se ao fato de que fatores sociodemográficos e ambientais tendem a ser correlacionados. ${ }^{20}$ De acordo com a autocorrelação espacial, ${ }^{13}$ regiões vizinhas tendem a apresentar mais similaridades que regiões distantes. A agregação dos dados gera problemas estatísticos por conta da redução da variabilidade, repercutindo sobre as inferências. ${ }^{12}$ 
Nesse tipo de estudo os modelos apresentados para explicar os componentes da mortalidade infantil estão expressos como combinações lineares entre indicadores socioeconômicos e demográficos. No entanto, desfechos e covariáveis são tomados simultaneamente, portanto a relação de causa e conseqüência não está perfeitamente caracterizada, sendo preferível o termo "possíveis determinantes" para tais covariáveis. ${ }^{13}$

Outras limitações são impostas pelos indicadores incluídos na análise, levando-se em consideração a qualidade das informações disponibilizadas pelo Datasus e outros sistemas utilizados. Uma das possíveis causas para as diferenças encontradas entre os modelos de 1991 e 2000 está relacionada ao fato de o conjunto de preditores para cada ano não ser o mesmo por imposição dos próprios bancos de dados. Além disso, diferentes estruturas de correlação entre as variáveis disponíveis podem, por si só, levar a diferentes conjuntos de preditores estatisticamente significantes.

Apesar das limitações, existem muitas razões para o uso dos estudos ecológicos, dentre as quais citam-se o baixo custo por trabalhar, geralmente, com dados secundários; maior facilidade no trato de variáveis, quando existe limitação quanto à mensuração individual; interesse em efeitos ecológicos, a exemplo da avaliação de programas ou políticas; e por último o senso de oportunidade, levando em consideração o nível de desenvolvimento das técnicas de análise, orientadas ao manejo das dificuldades pertinentes ao modelo de estudo em questão.

Os coeficientes de determinação $\left(\mathrm{R}^{2}\right)$ apresentados traduzem que o conjunto das variáveis incorporadas nos modelos de regressão foram capazes de explicar cerca de $35 \%$ e $32 \%$ da variabilidade do log-neperiano da TMI em 1991 e 2000, respectivamente. O restante da variação deve-se a outros determinantes não contemplados nesses modelos.

No presente estudo, a relação entre TMI e saneamento em 1991 deu-se por meio de uma relação inversa entre a proporção de imóveis urbanos com abastecimento público de água e a TMI. Para 2000, com a ampliação desse serviço (cerca de $80 \%$ dos imóveis urbanos contavam com abastecimento público de água), essa relação reaparece pela proporção de imóveis ligados à rede de esgotos. Em 2000, menos de 30\% dos domicílios urbanos do Ceará estavam ligados à rede coletora, situação que pouco se alterou nos últimos anos.* A proporção de residências pequenas, encontrada no modelo de 1991, também reforça a aproximação entre mortalidade infantil e a precariedade das habitações nos grandes centros urbanos, idéia corroborada por Monteiro \& Nazário ${ }^{11}$ (2000) quando avaliou as condições ambientais da saúde na infância na cidade de São Paulo.
A taxa de fecundidade total foi um dos fatores constituintes do modelo explicativo da TMI para 1991. Segundo estudos de Patarra ${ }^{16}(2000)$ nas décadas de 80 e 90, a queda na taxa de fecundidade no Brasil contribuiu com o declínio da TMI e conseqüente melhora da esperança de vida. ${ }^{18}$ Confirma-se, assim, a presunção existente em torno do efeito da fecundidade, cuja diminuição influencia diretamente na queda da mortalidade infantil, fazendo com que se evitem exposições como a multiparidade, o curto intervalo interpartal e as idades de parto extremas. ${ }^{3}$ Essa tese é corroborada por Costa et $\mathrm{al}^{5}$ (2003) que consideram plausível que a queda da fecundidade para o Brasil na década de 80 foi a principal responsável pela manutenção do declínio da mortalidade infantil. Tal fator não se tornou evidente em 2000, provavelmente, por sua estabilização ao final da década.

Outro preditor destacado em 1991 foi a taxa de urbanização. O caráter da relação direta se explica, possivelmente, pelas condições de vida adversas nas grandes cidades para a grande maioria dos imigrantes. Com baixa escolaridade e pouca ou nenhuma qualificação profissional, esses imigrantes formaram um enorme contingente populacional, vivendo com baixa renda, sem habitação adequada nem acesso a serviços básicos sanitários. ${ }^{3}$ No Ceará, as décadas de 70 e 80 foram caracterizadas pela busca de um novo perfil de sobrevivência por parte da população, desacreditada na agricultura e na pecuária de subsistência. Essa população encontra nos centros urbanos maiores os pólos industriais e comerciais, provocando grandes movimentos migratórios e o crescimento populacional desordenado dessas cidades, principalmente para a capital do estado.

Variáveis indicadoras de baixas condições de vida foram preditoras em 1991, persistindo sua determinação em 2000. São indicadores de vulnerabilidade e renda e se encontraram diretamente relacionados à TMI em 2000: a proporção de crianças de dez a 14 anos que trabalham, a proporção de chefes de família com renda mensal menor que $1 / 2$ salário mínimo, ambas em 1991, e a intensidade da pobreza. No entanto, discutem-se duas particularidades. A primeira é relativa àqueles indicadores de renda média, tal como o produto interno bruto (PIB) per capita, que podem provocar um viés de interpretação na relação com a mortalidade infantil, pois mesmo que o município apresente nível satisfatório, isso não implicará, necessariamente, em distribuição de renda. A segunda particularidade refere-se ao fato de que a melhoria nas condições econômicas de um município somente é traduzida em ganhos sociais se favorecem investimentos nos demais setores tais como educação, saúde, acesso a bens e serviços, habitação, e outros.

* Fundação Instituto Brasileiro de Geografia e Estatística. Departamento de População e Indicadores Sociais. Síntese de indicadores sociais 2002. Rio de Janeiro; 2002. 
Embora tenha havido redução da taxa de atividade no grupo etário de dez a 14 anos em todas as regiões brasileiras, o Nordeste ainda concentra a maior parcela de crianças em atividade.* Em 1997 e 2001, o Ceará atingiu a marca de $23,4 \%$ e $19,0 \%$, respectivamente. Na região Sudeste, esses valores são de 10,8\% em 1997, declinando para $8,1 \%$ em 2001. A redução da participação de crianças e adolescentes na constituição da população economicamente ativa deve estar associada a uma melhoria na educação dos jovens.*

Tomando por base o PIB per capita, o Ceará apresentou uma série que variou de R\$2.418,07, em 1991, a 2.794,14, em 2000, abaixo da média regional de $\mathrm{R} \$$ $3.014,15$, enquanto estados como São Paulo, Rio de Janeiro, Rio Grande do Sul e Distrito Federal detiveram, em 2000, um PIB per capita da ordem de R\$ 9.995,11, R\$ 9.571,49, R\$ 8.341,04 e R\$ 14.405,37, respectivamente.**

A diferença inter-regional quanto ao percentual da população em estado de pobreza (renda familiar mensal de até 1/4 salário mínimo), no período de 1992-2001, foi marcante. Baseando-se neste último ano, a região Nordeste apresentou $50,9 \%$ de sua população nessa condição, $* * *$ e o Ceará teve como resultado $52,1 \%$.

Os indicadores de nível educacional estão contemplados nos modelos de regressão de ambos os períodos. É reconhecido pelo Banco Mundial que as políticas de desenvolvimento educacional são fortemente aliadas dos ganhos obtidos em saúde. O grau de instrução é considerado um marcador da condição socioeconômica da mãe e de sua família. Além desse enfoque, o nível educacional da mãe pode ser compreendido também como fator relacionado ao perfil cultural e a comportamentos ligados aos cuidados de saúde, que têm um importante efeito na determinação da mortalidade infantil. ${ }^{7}$

A presença da proporção de crianças menores de dois anos desnutridas e da proporção do valor da produção vegetal em relação ao total do estado no modelo explicativo da TMI, em 2000, reforça a importância da relação entre alimentação e mortalidade infantil. Com isso, supõe-se que municípios com maior produção agrícola revertam essa produção, em parte, para sua própria população. Na composição do PIB cearense de 2000, observa-se um maior crescimento da agropecuária frente aos demais componentes, tomando-se como referência os anos de 1998 e 1999.**** Alimentos, ambiente e cuidados à criança estão condicionados pelo nível de renda, embora possam ser modificados por ações como serviços públicos de saúde, saneamento, educação e outras de caráter compensatório. ${ }^{4}$
O índice de envelhecimento, contido no modelo de 2000, mostra a importância dos recursos provenientes das aposentadorias em municípios de extrema pobreza ${ }^{15}$ e o envelhecimento da população brasileira ao longo das últimas décadas, processo denominado de transição demográfica. ${ }^{16}$ A proporção de crianças e jovens que até a década de 1970 era de $42,1 \%$ vem declinando, chegando a 2000 com $29,6 \%$ do total. Estimativas apontam a continuação da redução da participação de crianças e jovens $(23,3 \%)$ e a manutenção da tendência do aumento de idosos $(12,6 \%)$ até 2020 , ou seja, um incremento relativo de $47 \%$ nos próximos 20 anos. $^{6}$

A proporção de despesa com recursos humanos em relação à despesa total em saúde, encontrada no modelo de 2000, revela a importância dos investimentos em pessoal na melhoria da qualidade do nível de saúde, avaliada pela mortalidade infantil. Um exemplo concreto desse investimento, no Ceará, é o PSF, que gerou diversos postos de trabalho para profissionais de saúde, criando a necessidade de uma rede ambulatorial e hospitalar hierarquizada e de um corpo clínico qualificado que garanta atenção secundária nas diferentes regiões do estado. ${ }^{1}$

Renda, educação e saneamento se mantiveram como possíveis determinantes da TMI. Entretanto foram introduzidas novas variáveis na análise de 2000, possibilitando que elementos ligados a atenção à saúde, a produção agrícola, ao envelhecimento da população e a distribuição de renda passassem a explicar o comportamento da TMI. A urbanização, a fecundidade e o trabalho infantil saíram do modelo.

Após a adoção das medidas públicas de atenção seletiva à saúde da criança, observou-se uma efetiva redução na TMI na década de 90, em todos os seus municípios. Todavia, isso não significa que os determinantes socioeconômicos perderam sua importância em relação ao favorecimento a sobrevivência infantil. Na ausência de transformações sociais mais profundas, o que passa a distinguir os municípios quanto à redução na TMI, é a maior intensidade na aplicação dessas medidas seletivas de atenção primária por parte dos gestores estadual e municipais.

Essas estratégias de atenção básica são medidas emergenciais e insuficientes à redução da mortalidade infantil e à sua manutenção em nível mínimo possível. ${ }^{5}$ Sua continuidade dependerá de transformações estruturais, como: melhor distribuição de renda pela geração de empregos e estímulo à produção, acesso da população aos dividendos dessa produção, inclusão

\footnotetext{
* Fundação Instituto Brasileiro de Geografia e Estatística. Departamento de População e Indicadores Sociais. Síntese de indicadores sociais 2002. Rio de Janeiro; 2002.

** Ministério da Saúde. Departamento de Informática do Sistema Único de Saúde. Disponível em www.datasus.gov.br. Brasília; 2003.

**** Ministério da Saúde. Departamento de Informática do Sistema Único de Saúde. Disponível em www.datasus.gov.br. Brasília; 2004.

**** Frota JPH. Situação atual e estratégias para o desenvolvimento do estado do Ceará [palestra realizada no Sistema FIEC em 15.01.2001]. [acesso em 10/1/2003]. Disponível em: http://www.sfiec.org.br/palestras/default.htm 2001
} 
da população em programas educacionais, expansão do saneamento básico e da atenção à saúde em níveis primário e secundário. Essas medidas são exemplos de ações institucionais concretas que afetam os níveis de mortalidade infantil e redimensionam a ordem de seus determinantes.

\section{REFERÊNCIAS}

1. Andrade FM, editor. O programa de saúde da família no Ceará. Fortaleza: Expressão Gráfica e Editora; 1998. Ceará: abrindo as portas para saúde dentro de casa;p.49-59.

2. Arroyo P, Langer A, Avila H, Llerena C. Modelo para el analisis de la sobrevivencia en la infancia. Salud Publica Mex. 1988;30(3):463-9.

3. Ayçaguer LCS, Duran Macho E. Mortalidad infantil y condiciones higienico-sociales en las Ameritas: un estudio de correlación. Rev Saude Publica. 1990;24(6):473-80.

4. Benício MH, Monteiro CA, Costa-Rosa TE. Evolução da agenda tradicional da saúde pública: deficiências nutricionais. Evolução da desnutrição, da pobreza e do acesso a serviços públicos em dezesseis estados. In: Monteiro CA, organizador. Velhos e novos males da saúde no Brasil: a evolução do país e de suas doenças. 2. ed. São Paulo: Hucitec; 2000. p. 141-52.

5. Costa MCN, Mota ELA, Paim JS, Silva LMV, Texeira MG, Mendes CMC. Mortalidade infantil no Brasil em períodos recentes de crise econômica. Rev Saude Publica. 2003;37(6):699-706.

6. Fundo das Nações Unidas para a Infância. Situação da infância brasileira: 2001. Brasília: 2001.

7. Goldani MZ, Benatti R, Silva AAM, Bettiol H, Correa JCW, Tietzmann M, et al. Narrowing inequalities in infant mortality in Southern Brazil. Rev Saude Publica. 2002;36(4):478-83.

8. Hamilton LC, editor. Statistics with STATA. Belmont: Duxbury Thompsom Learning; 2003. Regression diagnostics. p. 152-70.

9. Hosmer DW, Lemeshow S, editors. Applied logistic regression. New York: Wiley; 1989. Model-building strategies and methods for logistic regression. p. 83133.

10. Monteiro CA, Nazário CL. Evolução da agenda tradicional da saúde pública: mortalidade infantil e materna. Declínio da mortalidade infantil e eqüidade social: o caso da cidade de São Paulo entre 1973 e 1993. In: Monteiro CA, organizador. Velhos e novos males da saúde no Brasil: a evolução do país e suas doenças. 2.ed. São Paulo: Hucitec; 2000. p. 173-85.
Com o aumento relativo do componente neonatal da mortalidade infantil, outras medidas diferentes da atenção primária necessitam ser desenvolvidas. A redução da mortalidade neonatal está também na dependência de uma assistência de maior qualidade ao pré-natal, ao parto e ao recém-nascido.

11. Monteiro CA, Nazário CL. Evolução de condicionantes ambientais da saúde na infância na cidade de São Paulo (1984-1996). Rev Saude Publica. 2000;34(6 supl.):13-8.

12. Morgenstern H. Ecologic studies in epidemiology: concepts, principles and methods. Ann Rev Public Health. 1995;16:61-81.

13. Morgenstern H. Ecologic studies. In: Rothman K, editor. Modern epidemiology. Philadelphia: Lippincott; 1998. p. 459-80.

14. Mosley WH. Determinantes biologicos y socioeconomicos de la sobrevivencia en la infancia. Salud Publica Mex. 1988;30(3):312-28.

15. O Globo (editorial). O fôlego da terceira idade: segurados do INSS respondem por até $60 \%$ do movimento de pequenos mercados no interior. $\mathrm{O}$ Globo. Rio de Janeiro, 2004 nov 7: 33

16. Patarra NL. Mudanças em condicionantes da saúde. Mudanças na dinâmica demográfica. In: Monteiro CA organizador. Velhos e novos males da saúde no Brasil: a evolução do país e de suas doenças. 2. ed. São Paulo: Hucitec; 2000. p. 61-8.

17. Population Reference Bureau. Cuadro de la poblacion mundial: datos e cálculos demográficos sobre los países y regiones del mundo. Washington: 2001.

18. Simões CCS, Ortiz LP. A mortalidade infantil no Brasil nos anos 80. In: Chadad J, Cervini R, editores. Crise e infância no Brasil: o impacto das políticas de ajustamento econômico. São Paulo: IPE/USP; 1988. p. 243-68.

19. Simões CCS. Perfis de saúde e de mortalidade no Brasil: uma análise de seus condicionantes em grupos populacionais específicos. Brasília: OPAS; 2002.

20. Susser M. The logic in ecological: II. the logic of design. Am J Public Health. 1994;84(5):830-5.

21. Szwarcwald CL, Bastos Fl, Andrade CLT. Medidas de desigualdad en salud: la discusión de algunos aspectos metodológicos con una aplicación para la mortalidad neonatal en el Municipio de Rio de Janeiro, 2000. Cad Saude Publica. 2002;18(4):959-70. 